\title{
Exclusion Principle Repulsion Effects on the Covalent Bond Beyond the Born-Oppenheimer Approximation
}

\author{
A. Sarsa, ${ }^{* a}$ J.M Alcaraz-Pelegrina, ${ }^{b \neq}$ and C. Le Sech ${ }^{c}$
}

Received Date

Accepted Date

DOI: 10.1039/xxxxxxxxxx www.rsc.org/journalname

\begin{abstract}
The changes in the covalent bond of the hydrogen molecule limited in the space by a spherical hard boundary are studied. The sphere is moved along an axis parallel or orthogonal to the molecular axis. The diffusion Monte Carlo approach is used to solve the Schrödinger equation with the relevant boundary conditions and to evaluate the changes in the bond energy versus the location of the sphere. The vertical and lateral quantum forces exerted on the sphere are evaluated by calculating the energy derivative versus the distances to the sphere. The results show that the quantum forces present an important dependence with the distance and vanish rapidly as the separation between the sphere and the molecule increases. In the limiting case the molecular bond becomes broken due to the electronic depletion induced in the covalent bond. An application of this study is the modelisation of the forces exerted on the passivated cantilever of an Atomic Force Microscope probing the electron cloud in the contact mode in the Pauli exclusion regime.
\end{abstract}

\section{Introduction}

In a free isolated molecule the electrons are allowed to span all the space, but when atoms or molecules are present in the surroundings the well known coulomb forces between the charged particles of the system induce changes in the state, as for example the polarization. Another situation of interest is when two atoms come very close together, the overlap of the wave functions increases and a strong repulsion force appears. Atoms therefore occupy a volume and cannot be squeezed too closely together. This interaction is similar to a hard wall repulsion. This force has a quantum mechanical character rooted in the Pauli exclusion principle, and is often called the Exclusion Principle Repulsion. The celebrated term $1 / r^{12}$ in the Lennard-Jones potential accounts empirically for this strong repulsive force appearing when the atoms become separated by short distances of the order of a few Bohr radius, $a_{0}$. Even inside homopolar molecules, a pure covalent bond can be modified by Pauli repulsion due to steric constraints inside the molecule on the bonding electrons ${ }^{1-3}$. A new state emerges with a component of ionic bonding resulting from the electron depletion induced by Pauli repulsion. This charge-shifted mechanism accounts for the resonant energy between covalent and ionic states, which becomes a major part of the bonding.

\footnotetext{
a Departamento de Física, Campus de Rabanales Edif. C2, Universidad de Córdoba, E-14071 Córdoba, Spain; E-mail: fa1sarua@uco.es

${ }^{b}$ Departamento de Física, Campus de Rabanales Edif. C2, Universidad de Córdoba, E-14071 Córdoba, Spain

${ }^{c}$ Institut des Sciences Moléculaires d'Orsay (ISMO), CNRS, Univ. Paris-Sud, Université Paris-Saclay, F-91405 Orsay, France
}

Pauli exclusion principle contributes in an important way to the stability of the matter. For example, Pauli repulsion force overcomes gravity for star masses below the Chandrasekar limit leading to the formation of white dwarfs while for higher masses avoids gravitational collapse giving rise to neutron stars. Authoritative reviews devoted to this principle ${ }^{4,5}$ are recommended for further details and analysis. Effects of Pauli repulsion force are very important in other fields as for example in Nuclear Physics where it leads to a significant reduction of the tunneling probability hindering the fusion rates of atomic nuclei ${ }^{6}$. Pauli repulsion force is also the reason for the high resolution achieved with the use of noncontact Atomic Force Microscopy (AFM) when then microscope's tip apex is functionalized with $\mathrm{CO}$ molecules ${ }^{7,8}$. Vertical and lateral forces appear when manipulating individual atoms and molecules by scanning probe microscocopy, playing a major role when atoms move on metal surfaces ${ }^{9}$.

In principle, exchange forces between the atom and its surroundings could be accounted by employing completely antisymmetric state functions including the electrons of both subsystems ${ }^{10,11}$. This scheme may pose important computational complications. Besides the contribution of exchange forces due to the Pauli's principle for electrons of different atoms will be entangled with other forces, as coulomb repulsion or exchange within the target molecule. Therefore, changes induced in the molecule under consideration by the exclusion principle repulsion are not easy to isolate.

Confinement effects can be studied theoretically by placing the atom inside a closed cavity described in terms of a potential. Dif- 
ferent works have been carried out to analyse the change of the atomic and molecular properties as a function of the confinement, see e.g. ${ }^{12-21}$. Less is known for other conditions of spatial restriction in which a particular region of the space is forbidden ${ }^{22,23}$. This situation is found when a large molecule is close to another molecule giving rise to a restriction of the space available to the bonding electrons due to the exclusion principle. A physical example for this is provided by high resolution AFM images with cantilever's deflection at the picometre scale, where rapidly varying interatomic forces are of critical importance in the measurement process ${ }^{24}$. Another example is given by the supramolecular cavities that accelerate some chemical reactions ${ }^{25,26}$ and constitute a model to study the chemical activity of the enzymes.

In this work we study the effects of Pauli exclusion principle repulsion on the covalent bond beyond the Born-Oppenheimer approximation. We have considered the problem of the $\mathrm{H}_{2}$ molecule with one surrounding atom. A hard wall spherical potential centred at different distances from the molecule is used as a model for the surrounding atom. In this scheme Pauli exclusion Principle Repulsion is a constraint to the space available for the bonding electrons. We study the effect of the induced depletion in the electron cloud of the sigma covalent bond. Vertical and lateral forces have been obtained by solving the quantum mechanical problem for different geometries and distances. A non-adiabatic approach, including both nuclear and electronic motion is employed for the $\mathrm{H}_{2}$ molecule. The Diffusion Monte Carlo method is employed to carry out the calculations.

\section{Methodology}

The Hamiltonian for the $\mathrm{H}_{2}$ molecule is written in terms of Jacobi relative coordinates, see Fig. 1, by using a similar scheme as ${ }^{18}$

$$
H=-\frac{\hbar^{2}}{2 \mu} \frac{d^{2}}{d Z^{2}}-\frac{\hbar^{2}}{2 \varepsilon} \nabla_{\vec{r}_{1}}^{2}-\frac{\hbar^{2}}{2 \varepsilon} \nabla_{\vec{r}_{2}}^{2}+V\left(Z, \vec{r}_{1}, \vec{r}_{2}\right)
$$

where $Z$ is the internuclear separation, $\vec{r}_{i}$ is the position vector of the ith-electron with respect to the centre of mass of the nuclei and $V$ is the interaction potential. The mass polarization term has been neglected and $\mu$ and $\varepsilon$ are the reduced masses

$$
\varepsilon=\frac{2 m_{e} M_{p}}{m_{e}+2 M_{p}}, \quad \mu=\frac{M_{p}}{2} .
$$

We have used the following values for the proton mass, $M_{p}=$ $938.272029 \mathrm{MeV} / c^{2}$ and the electron mass $m_{e}=0.5109989 \mathrm{MeV} / c^{2}$ obtaining $\varepsilon=0.9997278$ and $\mu=918.0763$ in atomic units. In the non-adiabatic scheme of this work the nuclei are allowed to vibrate and the direction of the molecular axis is kept fixed.

The interaction potential is written as

$$
V=V_{\text {coul }}+V_{\text {excl }}
$$

with $V_{\text {coul }}$ the coulomb interaction between electrons and nuclei, in atomic units,

$$
V_{\text {coul }}=-\frac{1}{r_{1 \mathrm{~A}}}-\frac{1}{r_{1 \mathrm{~B}}}-\frac{1}{r_{2 \mathrm{~A}}}-\frac{1}{r_{2 \mathrm{~B}}}+\frac{1}{r_{12}}+\frac{1}{Z}
$$

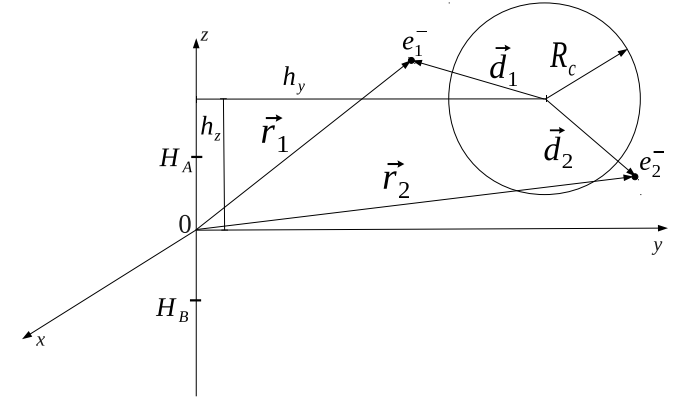

Fig. $1 \mathrm{H}_{2}$ molecule with an impenetrable spherical volume of radius $R_{c}$. The coordinates of the centre of the sphere are $\left(h_{x}=0, h_{y}, h_{z}\right)$.

where

$$
r_{12}=\left|\vec{r}_{1}-\vec{r}_{2}\right|, \quad r_{i \mathrm{~A}}=\left|\vec{r}_{i}-\hat{k} \frac{Z}{2}\right|, \quad r_{i \mathrm{~B}}=\left|\vec{r}_{i}+\hat{k} \frac{Z}{2}\right| \quad i=1,2
$$

where $\hat{k}$ is the direction of the molecular axis.

The potential, $V_{\text {excl }}$, is introduced to account for the fact that electrons cannot penetrate into a spherical domain of radius $R_{C}$ centred at

$$
\vec{h}=\left(0, h_{y}, h_{z}\right)
$$

We have considered $R_{c}=1 a_{0}$ in our calculations. Then, $V_{\text {excl }}$, can be written as

$$
V_{\mathrm{excl}}\left(\vec{r}_{1}, \vec{r}_{2}\right)=v_{\mathrm{excl}}\left(d_{1}\right)+v_{\mathrm{excl}}\left(d_{2}\right)
$$

where

$$
d_{i}=\left|\vec{d}_{i}\right|=\left|\vec{r}_{i}-\vec{h}\right|
$$

and

$$
v_{\text {excl }}(d)=\left\{\begin{array}{lll}
0 & \text { if } & d>R_{c} \\
\infty & \text { if } & d \leq R_{c}
\end{array}\right.
$$

Variational and Difussion Monte Carlo methods are employed to solve the time independent Schrödinger equation

$$
H \Psi\left(Z, \vec{r}_{1}, \vec{r}_{2}\right)=E \Psi\left(Z, \vec{r}_{1}, \vec{r}_{2}\right)
$$

with $H$ given in eqn (1). These methodologies have been reviewed in the literature, see for example ${ }^{27}$. The starting point is the Variational Monte Carlo (VMC) method. This technique is based on the Variational approach with the expectation value of the Hamiltonian calculated by using stochastic integration. As no analytic integration is done, very general forms of the trial wave function can be employed. Variational parameters are introduced in the trial wave function and the expectation value of the Hamiltonian is optimised with respect to them. The energy obtained is an upper bound to the exact energy of the ground state.

The optimised trial wave function is employed as guiding function in a Diffusion Monte Carlo (DMC) calculation. This method provides the ground state energy of a quantum many body system by using random walks to solve the Schrödinger equation. DMC exploits the formal analogy between a classical diffusion equation and the Schrödinger equation written in imaginary time. A short imaginary time approximation is employed to obtain an explicit expression of the Green's Function. A stochastic simulation of the 
different terms in the equation (diffusion plus first-order rate) is carried out. In the asymptotic limit of imaginary time, stationary behaviour is achieved and the ground state energy can be estimated. In practice, it is very important to use a guiding function to reduce the statistical uncertainties in the simulation. As the nodal surface is known exactly for the states here studied, we use the fixed-node DMC method that provides the exact ground state energy within the numerical error.

The wave function of this work is written as the product of different factors containing variational parameters

$$
\Psi_{t}\left(Z, \vec{r}_{1}, \vec{r}_{2}\right)=\Phi\left(Z, \vec{r}_{1}, \vec{r}_{2}\right) F(Z) J\left(r_{12}\right) w_{\mathrm{excl}}\left(d_{1}\right) w_{\mathrm{excl}}\left(d_{2}\right)
$$

This form has been previously employed ${ }^{18,20}$ to describe a confined $\mathrm{H}_{2}$ molecule beyond the Born-Oppenheimer approximation.

The function, $\Phi$, in eqn (10) is taken as

$$
\Phi\left(Z, \vec{r}_{1}, \vec{r}_{2}\right)=\phi\left(Z, \vec{r}_{1}\right) \phi\left(Z, \vec{r}_{2}\right)
$$

with

$$
\phi\left(Z, \vec{r}_{i}\right)=\exp \left[-(\alpha+\beta Z) \xi_{i}\right] \cosh \left\{[\alpha+(1-\beta) Z] \eta_{i}\right\}
$$

where and $\alpha$ and $\beta$ are variational parameters and $\xi_{i}$ and $\eta_{i}$ are confocal elliptic coordinates of the electron $i$

$$
\begin{aligned}
\xi_{i} & =\frac{r_{i \mathrm{~A}}+r_{i \mathrm{~B}}}{Z}, \quad 1 \leq \xi_{i} \leq \infty \\
\eta_{i} & =\frac{r_{i \mathrm{~A}}-r_{i \mathrm{~B}}}{Z}, \quad-1 \leq \eta_{i} \leq 1
\end{aligned}
$$

The factor $F(Z)$ accounts for nuclear vibration. We use the analytic form of the ground state wave function of the one dimensional harmonic oscillator

$$
F(Z)=e^{-\delta\left(Z-Z_{0}\right)^{2}}
$$

with $\delta$ and $Z_{0}$ variational parameters.

The function $J\left(r_{12}\right)$ is the electronic correlation factor. The following form has been used

$$
J\left(r_{12}\right)=\exp \left(\frac{b r_{12}}{1+c r_{12}}\right)
$$

with $b$ and $c$ variational parameters.

Finally the cut-off factors, $w_{\text {excl }}(d)$, account for the boundary condition imposed by the spatial restriction considered in this work, eqn (7) and (8). These functions are parameterized here as

$$
w_{\text {excl }}(d)=1-e^{-a_{c}\left(d-R_{c}\right)}
$$

with $a_{c}$ a variational parameter.

The variational parameters in the trial wave function of eqn (10) are $\alpha, \beta, \delta, Z_{0}, b, c$ and $a_{c}$. The variational energy is obtained for each case here studied from the minimization of the expectation value of the Hamiltonian by using the VMC method

$$
E_{\mathrm{VMC}}=\frac{\left\langle\Psi_{t}|H| \Psi_{t}\right\rangle}{\left\langle\Psi_{t} \mid \Psi_{t}\right\rangle}
$$

The optimal wave function is used in a DMC calculation to obtain the exact energy. We have employed three different time step values, $0.0005,0.0003$ and 0.0001 in a.u., for the systems here studied. All the calculations have been done by using 2000 walkers. A blocking scheme with 100 blocks is employed with 100000 steps per block. Before the calculation is started, a run of two blocks is carried out to equilibrate the initial ensemble of walkers. The statistical error is estimated as the standard deviation of the mean over the blocks. The Metropolis acceptance probability of the DMC calculation with the importance sampling of this work is above $99.997 \%$ for the time step values here considered. The mixed and growth energies agree within the numerical error. Extrapolated energies at zero time step are reported. The extrapolated energy coincides with the energy obtained by using smallest time step within the statistical error.

\section{Results}

\subsection{Energy}

We first study the case when the impenetrable sphere is located in the direction of the molecular axis, $h_{y}=0$. Location of the sphere between the nuclei would lead to bond scission. In Table 1 we report the VMC and DMC energies in hartree $\left(E_{\mathrm{h}}\right)$ along with the expectation value of the nuclear distance calculated variationally. The trial wave function has been optimised for each distance.

Table $1 \mathrm{VMC}, E_{\mathrm{VMC}}$, and DMC, $E_{\mathrm{DMC}}$, energies of the $\mathrm{H}_{2}$ molecule when the impenetrable sphere lies in the direction of the molecular axis, $h_{y}=0$, for different $h_{z}$ values. In parentheses we show the statistical error of the Monte Carlo calculation

\begin{tabular}{llll}
\hline$h_{z}\left(a_{0}\right)$ & $E_{\mathrm{VMC}}\left(E_{\mathrm{h}}\right)$ & $E_{\mathrm{DMC}}\left(E_{\mathrm{h}}\right)$ & $\langle Z\rangle\left(a_{0}\right)$ \\
\hline 2.5 & $-1.01745(4)$ & $-1.0422(2)$ & $1.26728(2)$ \\
3.0 & $-1.10677(3)$ & $-1.1209(1)$ & $1.34570(2)$ \\
3.5 & $-1.13849(3)$ & $-1.1488(1)$ & $1.39893(2)$ \\
4.0 & $-1.14913(3)$ & $-1.1592(1)$ & $1.43183(2)$ \\
5.0 & $-1.15377(3)$ & $-1.1635(1)$ & $1.44706(2)$ \\
$\infty$ & $-1.15475(3)$ & $-1.1640(1)$ & $1.44818(2)$ \\
\hline
\end{tabular}

A good agreement between the VMC and DMC energies is obtained, showing the good performance of the variational ansatz here employed. The quality of the variational energy is reduced as the sphere approaches the molecule. More sophisticated parameterizations of the cut-off factor, taking into account the steep decrease in the charge density, will improve the upper bound specially for the smaller $h_{z}$ values considered in this work. However, as the DMC method provides the exact ground state energy, that calculation is not carried out here.

For distances larger than $\sim 5 a_{0}$, the effect of the sphere is very small, the non-adiabatic energy of the free $\mathrm{H}_{2}$ molecule is ${ }^{28}$ $-1.164025 E_{\mathrm{h}}$. For $h_{z}<4 a_{0}$, the energy increases very rapidly as the sphere approaches the molecule. For $h_{z}=2.5 a_{0}$, the energy is very close to the dissociation threshold. The expectation value of the internuclear distance decreases as the sphere approaches the molecule. This is due to the depletion of the charge density in the neighbourhood of the sphere, that leads to larger values of the electron density in the region between the nuclei, as it is illustrated in Fig. 2. This gives rise to a shorter internuclear distance. The overall molecular size is reduced and both, the electrostatic repulsion and the kinetic energy increase. This effect 
is stronger than the lowering of the electrostatic attraction and the molecular energy rises.

In Table 2 we present the results obtained for the energy when the sphere approaches the molecule in a direction orthogonal to the molecular axis. We have considered $h_{z}=0$.

Table $2 \mathrm{VMC}, E_{\mathrm{VMC}}$, and DMC, $E_{\mathrm{DMC}}$, energies of the $\mathrm{H}_{2}$ molecule when the impenetrable sphere lies in the direction orthogonal to the molecular axis, $h_{z}=0$, for different $h_{y}$ values. In parentheses we show the statistical error of the Monte Carlo calculation

\begin{tabular}{llll}
\hline$h_{y}\left(a_{0}\right)$ & $E_{\text {VMC }}\left(E_{\mathrm{h}}\right)$ & $E_{\text {DMC }}\left(E_{\mathrm{h}}\right)$ & $\langle Z\rangle\left(a_{0}\right)$ \\
\hline 2.2 & $-1.00805(4)$ & $-1.0331(1)$ & $1.49778(2)$ \\
2.5 & $-1.07666(4)$ & $-1.0940(1)$ & $1.44937(2)$ \\
3.0 & $-1.12783(3)$ & $-1.1398(1)$ & $1.42876(2)$ \\
3.5 & $-1.14550(3)$ & $-1.1554(2)$ & $1.42805(2)$ \\
4.0 & $-1.15129(3)$ & $-1.1611(1)$ & $1.42550(2)$ \\
5.0 & $-1.15336(3)$ & $-1.1635(1)$ & $1.42696(2)$ \\
\hline
\end{tabular}

A similar trend on the energy is obtained for this case, i.e. the energy increases as the sphere gets closer to the molecule. Approaching in the direction of the molecular axis is more efficient to rise the energy than in an orthogonal direction. This is illustrated in Fig. 3 where we plot the energy when the sphere centre is either at the $y$ or $z$ axis as a function of $h_{z}$, or $h_{y}$ respectively.

Contrary to the previous case, the averaged internuclear distance increases when the sphere approaches the molecule in the transverse direction. The position of the impenetrable sphere in this geometry leads to a depletion of the electronic charge in the internuclear region, see Fig. 2. The attractive electron-nuclear interaction is reduced in absolute value. As a consequence of this, the internuclear distance is larger and the molecular energy rises. As it is the case of the covalent bond, the energy increment is lower when $\langle Z\rangle$ increases than when it decreases.

We have studied the case of off-axis approach. In Table 3 we report the energy obtained for $h_{y}=2.5 a_{0}$ and different $h_{z}$ values. The same is done in Table 4 for fixed $h_{y}=3.0 a_{0}$.

Table $3 \mathrm{VMC}, E_{\mathrm{VMC}}$, and DMC, $E_{\mathrm{DMC}}$, energies of the $\mathrm{H}_{2}$ molecule for $h_{y}=2.5 a_{0}$ and different $h_{z}$ values. The distance $d$ between the sphere centre and the centre of mass of the nuclei is also shown. In parentheses we show the statistical error of the Monte Carlo calculation

\begin{tabular}{lllll}
\hline$h_{z}\left(a_{0}\right)$ & $d\left(a_{0}\right)$ & $E_{\mathrm{VMC}}\left(E_{\mathrm{h}}\right)$ & $E_{\mathrm{DMC}}\left(E_{\mathrm{h}}\right)$ & $\langle Z\rangle\left(a_{0}\right)$ \\
\hline 0.7 & 2.6 & $-1.08796(4)$ & $-1.1038(1)$ & $1.41378(2)$ \\
1.5 & 2.9 & $-1.11659(4)$ & $-1.1300(1)$ & $1.40554(2)$ \\
2.0 & 3.2 & $-1.13239(3)$ & $-1.1443(1)$ & $1.40568(2)$ \\
2.5 & 3.5 & $-1.14288(3)$ & $-1.1537(1)$ & $1.414862)$ \\
5.0 & 5.6 & $-1.15425(3)$ & $-1.1639(1)$ & $1.44520(2)$ \\
\hline
\end{tabular}

Table $4 \mathrm{VMC}, E_{\mathrm{VMC}}$, and DMC, $E_{\mathrm{DMC}}$, energies of the $\mathrm{H}_{2}$ molecule for $h_{y}=3.0 a_{0}$ and different $h_{z}$ values. The distance $d$ between the sphere centre and the centre of mass of the nuclei is also shown. In parentheses we show the statistical error of the Monte Carlo calculation

\begin{tabular}{lllll}
\hline$h_{z}\left(a_{0}\right)$ & $d\left(a_{0}\right)$ & $E_{\mathrm{VMC}}\left(E_{\mathrm{h}}\right)$ & $E_{\mathrm{DMC}}\left(E_{\mathrm{h}}\right)$ & $\langle Z\rangle\left(a_{0}\right)$ \\
\hline 0.7 & 3.1 & $-1.13137(3)$ & $-1.1434(1)$ & $1.41695(2)$ \\
1.5 & 3.4 & $-1.14028(3)$ & $-1.1511(1)$ & $1.40010(2)$ \\
2.0 & 3.6 & $-1.14529(4)$ & $-1.1558(1)$ & $1.41122(2)$ \\
2.5 & 3.9 & $-1.14932(3)$ & $-1.1595(1)$ & $1.43542(2)$ \\
5.0 & 5.8 & $-1.15395(3)$ & $-1.1639(1)$ & $1.43566(2)$ \\
\hline
\end{tabular}

The energy rises as the sphere approaches the molecule. However, the increase on the energy is not as sharp as in the previous cases. This is illustrated in Fig. 3 where the energy is plotted as a function of $h_{z}$ for the two fixed $h_{y}$ values considered. For similar values of the distance between the sphere centre and the centre of mass of the nuclei, the off-axis approach leads to a lower energy rise. For small $h_{z}$ values, the rate of the increment of the energy is reduced due to the symmetry of the electron charge around the $\mathrm{H}-\mathrm{H}$ centre of mass.

The expectation value of the internuclear separation decreases as the distance between the molecule and the impenetrable sphere decreases. This is the same behaviour found when the impenetrable sphere lies in the direction of the molecular axis. The quantitative effect on $\langle Z\rangle$ is smaller now because the increase of the density in the internuclear region is lower in the lateral approach and the distance between the molecule and the sphere is larger.

\subsection{Forces on the sphere due to charge depletion}

The change of the energy of the molecule induced by the spatial limitation to the electronic cloud, can be employed as a model to study the quantum force appearing when two atomic or molecular species approach as for example in an Atomic Force Microscope. In high resolution AFM imaging, a functionalized tip probes the atoms or molecules of the sample. At very short distances, the force between them is responsible for the sharp submolecular resolution ${ }^{29}$. In the contact mode, where the tip of the cantilever penetrates inside the charge distribution and probes the molecular orbitals, Pauli exchange forces are important. An estimation of the force of the tip on the molecule can be carried out starting from the results of our model. It is beyond the purpose of this work to develop a realistic model for the different forces that may arise such as long range forces, capillarity, van der Waals, etc. that depend on the nature of the sample and the tip. We will consider only the force due to the exclusion of a part of the space available for the electrons of the molecule.

\subsubsection{Axial and Transverse forces}

Here we consider two situations. In the first one, the tip lies in the direction of the molecular axis, $h_{y}=0$. The repulsive axial force on the sphere can be obtained from our calculations by using the Hellman-Feynman theorem

$$
\begin{aligned}
F_{a} & =-\left\langle\frac{\partial H}{\partial h_{z}}\right\rangle_{h_{y}=0} \\
& =-\left.\frac{\partial E}{\partial h_{z}}\right|_{h_{y}=0}
\end{aligned}
$$

The second situation is when the tip approaches the molecule in a direction perpendicular to the its axis with $h_{z}=0$. The trans- 

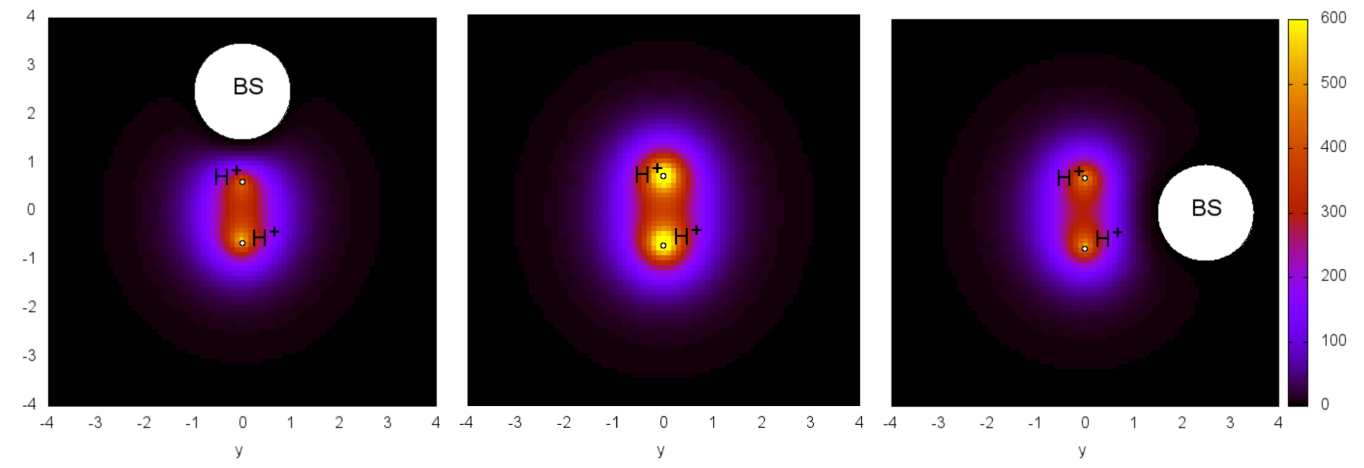

Fig. 2 Density maps of the electron density of the $\mathrm{H}_{2}$ molecule. Left hand side plot: the impenetrable sphere lies in the direction of the molecular axis; central plot: case without sphere; right hand side plot: the sphere lies in the direction orthogonal to the molecular axis.

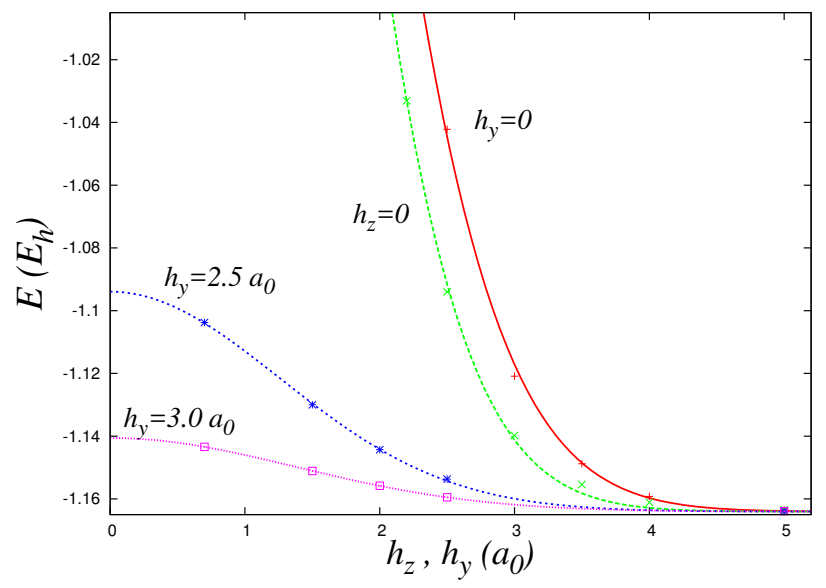

Fig. 3 Energy of the $\mathrm{H}_{2}$ molecule when the sphere is moved along the molecular axis as a function of $h_{z}$ for fixed $h_{y}$ values: $h_{y}=0, h_{y}=2.5 a_{0}$ and $h_{y}=3.0 a_{0}$; and as a function of $h_{y}$ for $h_{z}=0$.

verse force is calculated as

$$
\begin{aligned}
F_{t} & =-\left\langle\frac{\partial H}{\partial h_{y}}\right\rangle_{h_{z}=0} \\
& =-\left.\frac{\partial E}{\partial h_{y}}\right|_{h_{z}=0}
\end{aligned}
$$

The derivatives have been calculated by carrying out an analytic fit of the energy as a function of $h_{y}$ and $h_{z}$. In Fig. 4 we plot the axial and transverse and forces as a function of $h_{z}$ and $h_{y}$ respectively. The forces increase rapidly as the distance decreases between 3 and $2 a_{0}$. The force is more intense when tip approaches the molecule in the axial direction as compared to the transverse direction.

\subsubsection{Lateral forces}

Lateral forces have been calculated for off-axis approach for $h_{y}=$ $2.5 a_{0}$ and $h_{y}=3.0 a_{0}$ for different $h_{z}$ values by using the same scheme as before. In Fig. 4 we plot these lateral forces as a function of $h_{z}$. These forces are smaller than the axial and transverse ones. The changes in the electron density are less important in a lateral approach than when the impenetrable domain is in the

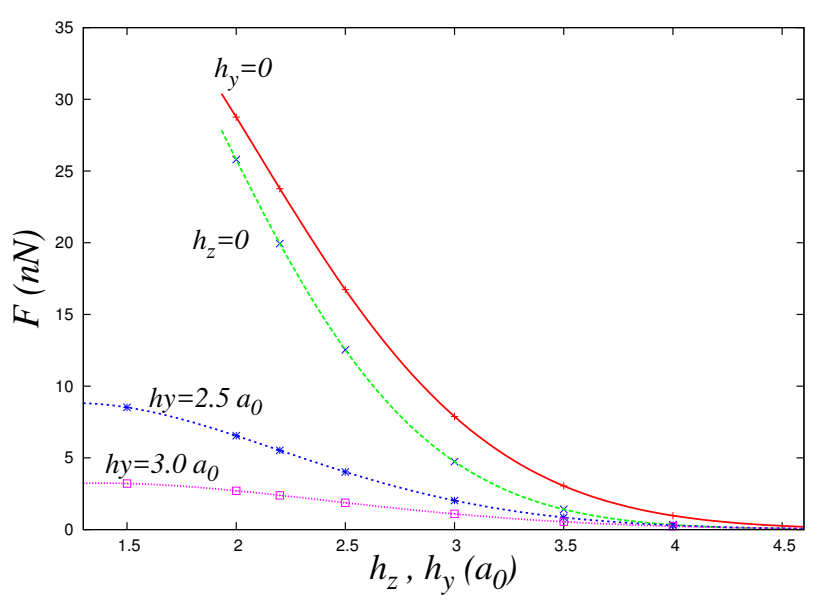

Fig. 4 Force exerted on the sphere as a function of $h_{z}$ for fixed $h_{y}$ values: $h_{y}=0, h_{y}=2.5 a_{0}$ and $h_{y}=3.0 a_{0}$; and as a function of $h_{y}$ for $h_{z}=0$.

axial or in the transverse directions.

\section{Conclusions}

Properties of a molecular system are modified when the space available to the electrons in the free state is restricted. The molecular binding energy is reduced due to the depletion of the electron density of the covalent bond. All these changes result fundamentally from the modification in the curvature of the wave function resulting from the wave nature of the particles.

In an AFM the tip of the cantilever, modeled by a hard sphere here, can induce such changes in the wave function in the Pauli's regime. Though the true nature of the force is not known in that case, the force can be estimated from the knowledge of the variation of the bond energy under the constraint. The results show an important dependence of the energy values and the vertical and lateral forces versus the distance between the bonding electron cloud and the hard sphere.

It might be of interest to note that, more generally, atoms in the close surrounding of the molecule can mimic the force due to the Pauli's principle and induce bond changes. This mechanism could be relevant in different situations when chemistry in confined spaces is considered. For example, inside a zeolite cav- 
ity, non-covalent interactions like Pauli repulsion can confine the substrate at the molecular level and influence the molecular orbitals ${ }^{30}$. Scanning tunneling microscopy can induce chemical reactions by the excitation of molecular states ${ }^{31-36}$. Bond scission is triggered by electric fields and tunneling currents. In our study we have considered only the effect of Pauli repulsion by the AFM tip, by using a hard wall spherical potential as a model for the surrounding atom. More sophisticated potential barriers can be implemented to mimic the effect of an electric field and to allow for the tunneling of the electrons.

\section{Acknowledgements}

This work was partially supported by the Spanish Dirección General de Investigación Científica y Técnica (DGICYT) and FEDER under contract FIS2015-69941-C2-2-P and the Junta de Andalucía under grant FQM378.

\section{References}

1 S. Shaik, D. Danovich, B. Silvi, D. Lauvergnat and P. C. Hiberty, Chem. Eur. J., 2005, 11, 6358-6371.

2 W. Wu, J. Gu, J. Song, S. Shaik and P. Hiberty, Angew. Chem. Int. Ed., 2009, 48, 1407-1410.

3 S. Shaik, D. Danovich, W. Wu and P. C. Hiberty, Nature Chem., 2009, 1, 443-449.

4 M. Massimi, Pauli's Exclusion Principle, Cambridge University Press, Cambridge, 2005.

5 S. Jarvis, A. Sweetmany, L. Kantorovichz, E. McGlynn and P. Moriarty, Imaging and Manipulation of Adsorbates using Dynamic Force Microscopy, Springer Verlag GMBH, Cham, Switzerland, 2015, vol. V, pp. 1-24.

6 C. Simenel, A. S. Umar, K. Godbey, M. Dasgupta and D. J. Hinde, Phys. Rev. C, 2017, 95, 031601.

7 L. Gross, F. Mohn, N. Moll, P. Liljeroth and G. Meyer, Science, 2009, 325, 1110.

8 L. Gross, Nature Chemistry, 2011, 3, 273.

9 M. Ternes, C. Lutz, C. Hirjibehedin, F. Giessibl and A. Heinrich, Science, 2008, 319, 1066.

10 J. Murrel, M. Randić, D. Williams and H. Longuet-Higgins, Proc. Roy. Soc. A, 1965, 284, 566-581.

11 J. Jensen and M. Gordon, Mol. Phys., 1996, 89, 1313-1325.

12 E. Ludeña, J. Chem. Phys., 1978, 69, 1770-1775.

13 E. Ley-Koo and S. A. Cruz, J. Chem. Phys., 1981, 74, 46034610.

14 R. LeSar and D. R. Herschbach, J. Phys. Chem., 1981, 85, 2798-2804.

15 J. Connerade, V. Dolmatov and P. Lakshmi, J. Phys. B., 2000, 33, 251.
16 R. Colín-Rodrígez, C. Díaz-García and S. A. Cruz, J. Phys. B: At. Mol. Opt. Phys., 2011, 44, 241001-1-8.

17 H. Montgomery Jr and K. D. Sen, Phys. Lett. A, 2012, 376, 1992-1996.

18 A. Sarsa, J. M. Alcaraz-Pelegrina, C. Le Sech and S. A. Cruz, J. Phys. Chem. B, 2013, 117, 7270-7276.

19 M. Rodríguez-Bautista, C. Díaz-García, A. Navarrete-López, R. Vargas and J. Garza, J. Chem. Phys, 2015, 143, 034103.

20 A. Sarsa, J. M. Alcaraz-Pelegrina and C. Le Sech, J. Phys. Chem. B, 2015, 119, 14364-14372.

21 E. Rashed and J. L. Dunn, Phys. Chem. Chem. Phys., 2019, 21, 3347-3359.

22 E. Ley-Koo, Avd. Quantum Chem., 2009, 57, 79-122.

23 A. Sarsa, J. M. Alcaraz-Pelegrina and C. Le Sech, Phys. Lett. A, 2017, 381, 780-786.

24 J. Sader, B. Huges, F. Huber and F. Giessibl, Nature Nanotechnology, 2018, 13, 1088-1091.

25 B. C. Pemberton, R. K. Singh, A. C. Johnson, S. Jockusch, J. P. Da Silva, A. Ugrinov, N. J. Turro, D. K. Srivastava and J. Sivaguru, Chem. Commun., 2011, 47, 6323-6325.

26 V. Sashuk, H. Butkiewicz, M. Fiałkowski and O. Danylyuk, Chem. Commun., 2016, 52, 4191-4194.

27 B. L. Hammond, W. A. J. Lester and P. J. Reynolds, Monte Carlo Methods in ab initio Quantum Chemistry, World Scientific, Singapore, 1994.

28 D. B. Kinghorn and L. Adamowicz, Phys. Rev. Lett., 1999, 83, 2541-2543.

29 P. Hapala, G. Kichin, C. Wagner, F. S. Tautz, R. Temirov and P. Jelínek, Phys. Rev. B, 2014, 90, 085421.

30 V. Mouarrawis, R. Plessius, J.I. van der Vlugt and J.N.H. Reek, Front. Chem., 2018, 6, 623.

31 H. Li, N. Kim, T. Su, M. Steigerwald, C. Nuckolls, P. Darancet, J. Leighton and L. Venkataraman, J. Am. Chem. Soc, 2016, 138, 16159-16164.

32 H. Li, T. Su, V. Zhang, M. Steigerwald, C. Nuckolls and L. Venkataraman, J. Am. Chem. Soc., 2015, 137, 5028-5033.

33 B. Borca, T. Michnowcz, R. Petuya, M. Pristl, V. Schendel, I. Pentegov, U. Kraft, H. Klauk, P. Wahl, R. Gutzer, A. Arnau, U. Schlickum and K. Kern, ACS Nano, 2017, 11, 4703-4709.

34 G. M. S.-W. Hla and K. Rieder, ChemPhysChem., 2001, 2, 361366.

35 G. Reecht, G. Lotze, D. Sysoiev, T. Huhn and K. Franke, J. Phys.: Condens. Matter, 2017, 29, 294001.

36 L. Zhang, E. Laborda, N. Darwish, B. Noble, J. Tyrell, S. Pluczyk, A. Brun, G. Wallace, J. González, M. Coote and S. Ciampi, J. Am. Chem. Soc., 2018, 140, 766-774. 